\title{
Endoscopic Marsupialisation of the Lateral Frontal Sinus Mucocele With Orbital Extension: A Case Report
}

\author{
Hadi Sharouny ${ }^{1, *} ;$ Prepageran Narayanan $^{2}$ \\ ${ }_{1}^{1}$ Department of Otorhinolaryngology Head and Neck Surgery, Faculty of Medicine, Shiraz University of Medical Sciences, Shiraz, IR Iran \\ ${ }^{2}$ Department of Otorhinolaryngology Head and Neck Surgery, Faculty of Medicine, University of Malaya, Kuala Lumpur, Malaysia \\ ${ }^{*}$ Corresponding Author: Hadi Sharouny, Department of Otorhinolaryngology Head and Neck Surgery, Faculty of Medicine, Shiraz University of Medical Sciences, Shiraz, IR Iran. Tel: \\ +98-7116291478, Fax:+98-7116291478, E-mail: hadi.sharouny@iran.ir.
}

Received: December 23, 2013; Revised: August 31, 2014; Accepted: October 23, 2014

\begin{abstract}
Introduction: Frontal sinus mucoceles are the commonest among all paranasal mucoceles. With introduction of functional endoscopic sinus surgery, surgeons prefer endoscopic management of sinus mucoceles, but lesions that appear in the lateral part of the frontal sinus can be difficult to access and often need external approaches.

Case Presentation: We described a lateral frontal sinus mucocele with intra-orbital extension, which was successfully managed by endoscopic sinus surgery.

Conclusions: Endoscopic sinus surgery is the treatment of choice in most frontal sinus mucoceles including lateral frontal mucoceles.
\end{abstract}

Keywords:Frontal Sinus; Mucocele; Endoscopy; Paranasal Sinus Diseases

\section{Introduction}

A mucocele is an epithelial lined, mucous containing sac completely filling the sinus and capable of expansion (1). The frontal sinus is said to be the most frequent, followed by the ethmoid sinus, the sphenoid sinus and maxillary sinus in the order of frequency (1-3). By limited fenestration of thin wall of mucoceles, they are drained into nasal cavity. If applied to mucoceles in the frontal bone, this procedure is called marsupialisation, which may require a broad endonasal frontal sinusotomy. If mucoceles are localized far laterally, an external approach operation of the frontal sinus might be necessary. We reported a case of lateral frontal sinus mucocele treated with endoscopic sinus surgery successfully.

\section{Case Presentation}

A 54-year-old man referred with complaints of swelling over right supra-orbital region and forward protrusion of the right eyeball for five months. The patient was admitted to University Malaya Medical Centre, in the city of Kuala Lumpur, Malaysia, in September 2012. He gave a history of double vision for three days and right frontal pain for five months. He did not have any history of previous trauma or sinus surgery. On examination, there was 2 by $3 \mathrm{~cm}$ single swelling extending superiorly to the supra-orbital rim, inferiorly $0.5 \mathrm{~cm}$ to the superior eyelid and medially to the medial canthus. Swelling was slightly tender, firm to hard, non- pulsatile, fixed to underlying structure, but overlying skin was free. Swelling was pushing the eyeball inferiorly, laterally and anteriorly (Figure 1). On ophthalmic examination, diplopia, right eye proptosis, limitation at upward gaze and hyperemia of the right optic disc were noted. Pupil was reactive to light. Sensation over cheek was intact. Anterior rhinoscopy and nasal endoscopy had normal findings. Computed tomography scan showed a well-defined homogenous soft tissue density in the lateral region of the right frontal sinus. Superior wall of sinus was intact, but floor of the frontal sinus was eroded and the mass extended into superomedial aspect of the orbit. The globe was displaced inferiorly and laterally by the mass (Figure 2). Based on clinical and radiological findings, diagnosis was likely to be mucocele. The diagnosis of right frontal mucocele was confirmed at surgery. Endoscopic anterior and posterior ethmoidectomies and frontal sinusotomy were performed under general anesthetic. The axillary flap approach was used to better access the frontal recess. The medial wall of the orbit (lamina papyracea) was perforated endoscopically, eyeball pushed laterally and the floor of the right frontal sinus removed. Mucocele was identified and marsupialised endoscopically. About 20 $\mathrm{mL}$ of thick yellow fluid was drained from the frontal sinus mucocele. Postoperatively, there was a rapid resolution of the swelling with obvious improvement of proptosis and eye movement (Figure 1). Patient was well in one month follow-up. 


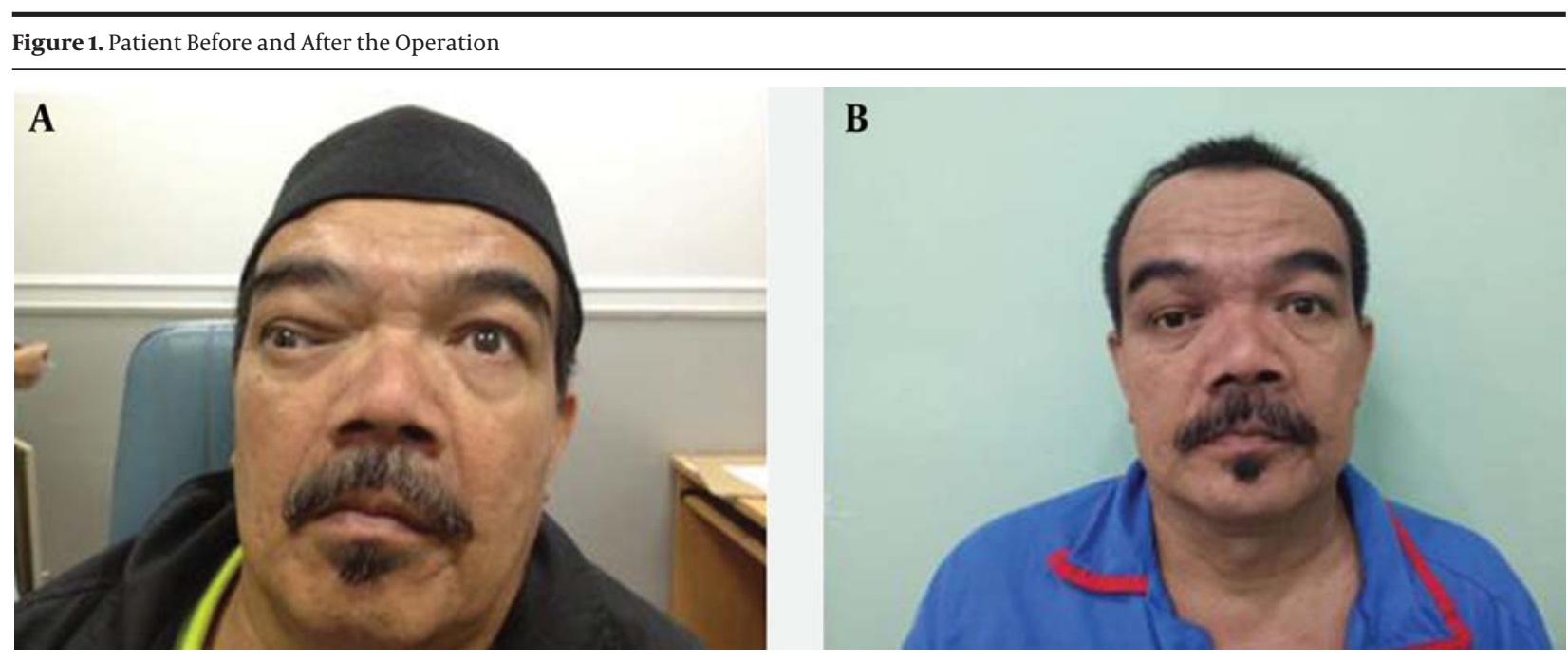

Left: Showing right supraorbital swelling and proptosis of the right eye. Right: Showing patient three days postoperatively.

Figure 2. Axial and Coronal Plane of CT Scan

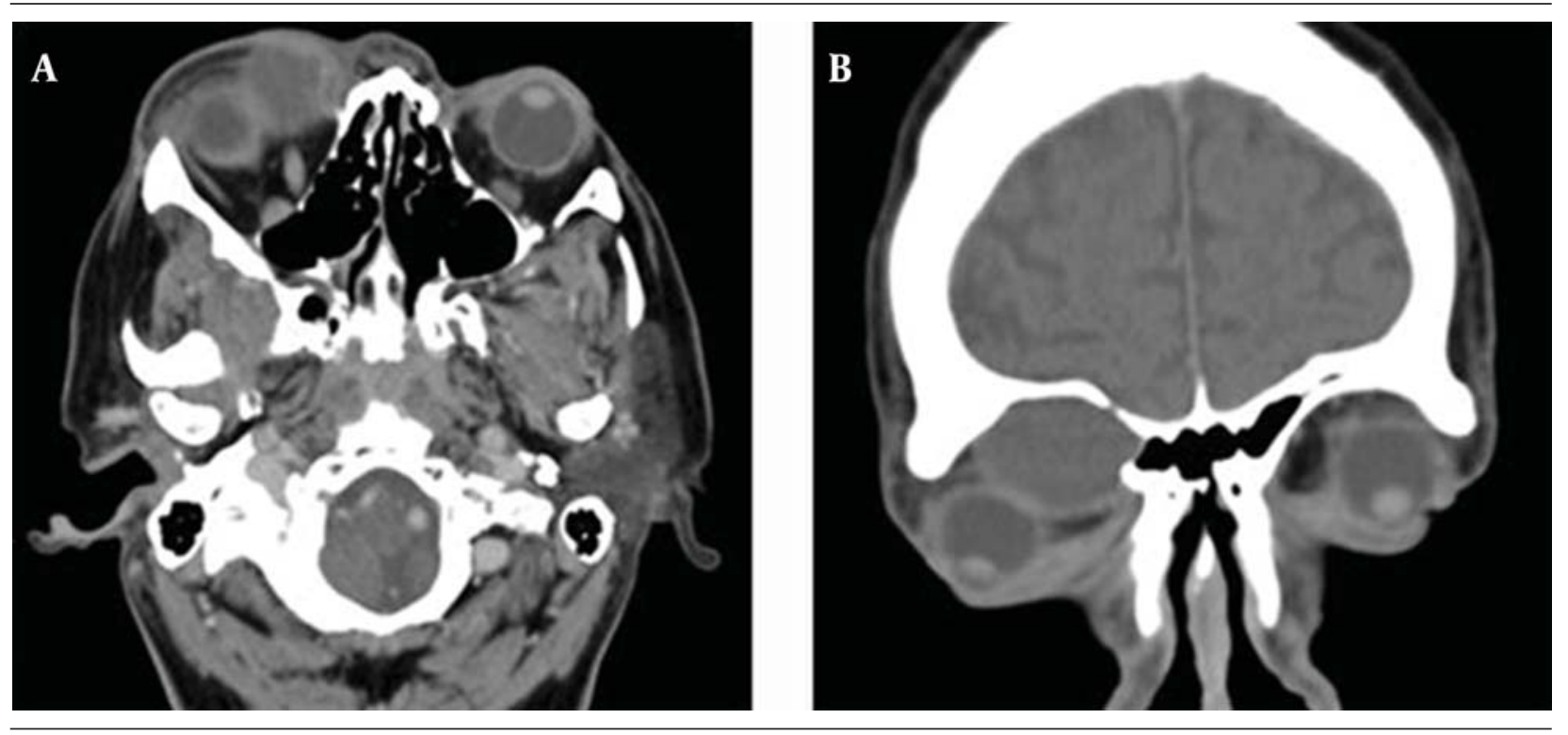

A, Axial plane of CT scan showing a well-defined homogenous mass pushing the right globe anteriorly. B, Coronal plane of CT scan showing the same mass eroded the right floor of frontal sinus and pushing the globe inferolaterally.

\section{Discussion}

Fronto-ethmoidal area is more susceptible to mucocele formation due to complexity of its drainage compared to other sinuses (1). There may be a considerable time lag between the initiating factor and clinical presentation of mucocele. In case of trauma, this is an average of 23 years, whereas following an acute infective episode, the mean time to presentation is 22 months (4). Clinical features of frontal mucocele are frontal headache, diplopia, proptosis, inferior displacement of globe, lateral displacement of globe, limited ocular mobility and reduced visual acuity $(3,5)$. Computed tomography (CT) scan is the op- timum method of demonstrating contents of mucocele (6). The appearances of mucocele contents on magnetic resonance imaging (MRI) vary considerably from low to high signal depending on the constituents of mucocele and generally not very helpful (3). Several treatment options are available and the choice depends on the degree of extension (2). The external approaches include a Lynch-Howarth frontoethmoidectomy or an osteoplastic flap (3). In most cases an endonasal endoscopic approach may be used (3). When appropriately selected, the advantages of endoscopic approach are lack of external scar, 
no disturbance of the trochlea and no supraorbital paresthesia. There would be significant reduction in orbital displacement. Endoscopic approach has the advantage of minimizing any double vision secondary to decompression (3). An endoscopic approach is ideal in those mucoceles which can be accessed and widely marsupialised. Therefore, virtually all ethmoid, maxillary and sphenoid mucoceles may be managed by entirely endoscopic approach (3). When the lesions coexist both intracranial and extracranial spaces, an intranasal approach should be selected as a first choice, because it is less invasive (2). The dura mater is considerably resistant to pressure or inflammation, which is rarely penetrated (7). In general, benign neoplasms of frontal sinus and tumors not extending more laterally than a vertical plane through lamina papyracea may be operated through the nose (8). In a retrospective study, 10 patients were identified with lateral frontal sinus mucoceles. These patients underwent surgical drainage using computer-aided endoscopic techniques. Initially, endoscopic drainage of mucocele was successful in all patients. One patient was lost to follow-up after three months and one patient underwent a revision endoscopic sinus surgery with trephination five months after the initial drainage. The remaining eight patients remained free of disease by nasal endoscopy and postoperative computer tomography scans (9). In a study performed on 10 cadaver heads, it was revealed that the Modified Lothrop Procedure (Draf III) could provide extended access to the frontal sinus. The study showed that endoscopic access to the lateral frontal sinus walls is excellent except for the sinus floor. For sinuses pneumatized laterally beyond the midorbital point, only $10 \%$ of lateral orbital roofs were accessible (10). In another study performed on three cadavers for unilateral frontal lesions, the Modified hemi-Lothrop Procedure was shown to provide adequate access to lateral frontal and distal supraorbital sinus diseases from the contralateral nasal cavity via a superior septectomy window (11). In our case, frontal mucocele was situated laterally, but by removing lamina papyracea, endoscopic operation was performed successfully. This case report introduced a new approach to lateral frontal sinus lesions such as mucoceles. In most cases, an endonasal endoscopic approach might be used for mucoceles (3). Considering the benign nature of mucocele, endoscopic approach with less complication is a better treatment even for patients with intracranial extension(2). The main disadvantage of the current method is possible extraocular muscle damage and strabismus. Since using microdebrider can cause irreparable muscle damage, it is not suggested in this method (12). From our point of view, most of frontal sinus mucoceles including lateral frontal mucoceles can be operated endoscopically and endoscopic sinus surgery is the treatment of choice for these lesions.

\section{Authors' Contributions}

Acquisition, analysis and interpretation of data and drafting of the manuscript: Hadi Sharouny. Study supervision: Prepageran Narayanan.

\section{References}

1. Natvig K, Larsen TE. Mucocele of the paranasal sinuses. A retrospective clinical and histological study. J Laryngol Otol. 1978;92(12):1075-82.

2. Koike Y, Tokoro K, Chiba Y, Suzuki SI, Murai M, Ito H. Intracranial extension of paranasal sinus mucocele: two case reports. Surg Neurol.1996;45(1):44-8.

3. Lund VJ. Mucoceles. In: Gleeson M, Browning GG, Burton MJ, Clarke R, Hibbert J, Jones NS editors. Scott-Brown's Otorhinolaryngology, Head and Neck Surgery: Hodder-Arnold; 2008. pp. 1531-8.

4. Lund VJ. Anatomical considerations in the aetiology of frontoethmoidal mucoceles. Rhinology. 1987;25(2):83-8.

5. Kuran G, Vuralkan E, Saka C, Akin I. Endoscopic sinus surgery of frontal mucocele. J Pak Med Assoc. 2007;57(11):570-1.

6. Kennedy DW, Josephson JS, Zinreich SJ, Mattox DE, Goldsmith MM. Endoscopic sinus surgery for mucoceles: a viable alternative. Laryngoscope. 1989;99(9):885-95.

7. Delfini R, Missori P, Iannetti G, Ciappetta P, Cantore G. Mucoceles of the paranasal sinuses with intracranial and intraorbital extension: report of 28 cases. Neurosurgery. 1993;32(6):901-6.

8. Draf W. The Frontal Sinus. In: Gleeson M, Browning GG, Burton MJ, Clarke R, Hibbert J, Jones NS editors. Scott-Brown's Otorhinolaryngology, Head and Neck Surgery: Hodder-Arnold; 2008. pp. 1500-30.

9. Chiu AG, Vaughan WC. Management of the lateral frontal sinus lesion and the supraorbital cell mucocele. Am J Rhinol. 2004;18(2):83-6.

10. Timperley DG, Banks C, Robinson D, Roth J, Sacks R, Harvey RJ. Lateral frontal sinus access in endoscopic skull-base surgery. Int Forum Allergy Rhinol.2011;1(4):290-5.

11. Eloy JA, Friedel ME, Murray KP, Liu JK. Modified hemi-Lothrop procedure for supraorbital frontal sinus access: a cadaveric feasibility study. Otolaryngol Head Neck Surg. 2011;145(3):489-93.

12. Thacker NM, Velez FG, Demer JL, Wang MB, Rosenbaum AL. Extraocular muscle damage associated with endoscopic sinus surgery: an ophthalmology perspective. Am J Rhinol. 2005;19(4):400-5. 